\title{
Equidistribution of phase shifts in trapped scattering
}

\author{
Maxime Ingremeau
}

March 23, 2022

\begin{abstract}
We consider semiclassical scattering for compactly supported perturbations of the Laplacian and show equidistribution of eigenvalues of the scattering matrix at (classically) non-degenerate energy levels. The only requirement is that sets of fixed points of certain natural scattering relations have measure zero. This extends the result of GRHZ15, where the authors proved the equidistribution result under a similar assumption on fixed points but with the condition that there is no trapping.
\end{abstract}

\section{Introduction}

Consider a Riemannian manifold $(X, g)$ which is Euclidean near infinity, in the sense that there exist compact sets $X_{0} \subset X$ and $K_{0} \subset \mathbb{R}^{d}$ such that $\left(X \backslash X_{0}, g\right)$ and $\left(\mathbb{R}^{d} \backslash K_{0}, g_{\text {eucl }}\right)$ are isometric.

Let us consider an operator $P_{h}:=-h^{2} \Delta_{g}+V$, where $V \in C_{c}^{\infty}(X)$ has its support in $X_{0}$. It is well-known (see for example [Mel95, §2] or [DZ, §3.7, §4.4]), that for any $\phi_{i n} \in C^{\infty}\left(\mathbb{S}^{d-1}\right)$, there is a unique solution to $\left(P_{h}-1\right) u=0$ satisfying, for all $x \in\left(X \backslash X_{0}\right) \cong\left(\mathbb{R}^{d} \backslash K_{0}\right)$ :

$$
u(x)=|x|^{-(d-1) / 2}\left(e^{-i|x| / h} \phi_{\text {in }}(\omega)+e^{i|x| / h} \phi_{\text {out }}(-\omega)\right)+O\left(|x|^{-(d+1) / 2}\right) .
$$

We define the scattering matrix ${ }^{1} S_{h}: C^{\infty}\left(\mathbb{S}^{d-1}\right) \longrightarrow C^{\infty}\left(\mathbb{S}^{d-1}\right)$, which depends on $h$, by

$$
S_{h}\left(\phi_{\text {in }}\right):=e^{i \pi(d-1) / 2} \phi_{\text {out }} .
$$

The factor $e^{i \pi(d-1) / 2}$ is taken so that the scattering matrix is the identity operator when $(X, g)=$ $\left(\mathbb{R}^{d}, g_{\text {Eucl }}\right)$ and $V \equiv 0$.

For each $h \in(0,1], S_{h}$ can be extended by density to a unitary operator acting on $L^{2}\left(\mathbb{S}^{d-1}\right)$. $S_{h}-I d$ is then a trace class operator. Therefore, $S_{h}$ admits a sequence of eigenvalues of modulus 1 , which converge to 1 , and which we denote by $\left(e^{i \beta_{h, n}}\right)_{n \in \mathbb{N}}$.

Our aim in this paper will be to study the behaviour of $\left(e^{i \beta_{h, n}}\right)$ in the limit where $h \rightarrow 0$. To do this, we define a measure $\mu_{h}$ on $\mathbb{S}^{1}$ by

$$
\left\langle\mu_{h}, f\right\rangle:=(2 \pi h)^{d-1} \sum_{n \in \mathbb{N}} f\left(e^{i \beta_{h, n}}\right),
$$

for any continuous $f: \mathbb{S}^{1} \longrightarrow \mathbb{C}$. This measure is not finite, but $\left\langle\mu_{h}, f\right\rangle$ is finite as soon as 1 is not in the support of $f$.

Let us now state the assumptions we make on the manifold $X$ and on the potential $V$.

\footnotetext{
${ }^{1}$ which is not a matrix as soon as $d>1$ !
} 
The scattering map We denote by $p(x, \xi)=|\xi|_{g}^{2}+V(x): T^{*} X \longrightarrow \mathbb{R}$ the classical Hamiltonian, which is the principal symbol of $P_{h}$. Let us write $\mathcal{E}$ for the energy layer of energy 1 :

$$
\mathcal{E}=\left\{(x, \xi) \in T^{*} X ; p(x, \xi)=1\right\} .
$$

We denote by $\Phi^{t}(\rho)$ the Hamiltonian flow for the Hamiltonian $p$. The outgoing and incoming sets are defined as

$$
\Gamma^{ \pm}:=\left\{\rho \in \mathcal{E}, \text { such that } \Phi^{t}(\rho) \text { remains in a compact set for all } \mp t \geq 0\right\} .
$$

The trapped set is the compact set

$$
K=\Gamma^{+} \cap \Gamma^{-} .
$$

Since, away from $X_{0}$, the trajectories by $\Phi^{t}$ are just straight lines, we have that for any $\omega \in \mathbb{S}^{d-1}$, and $\eta \in \omega^{\perp} \subset \mathbb{R}^{d}$, there exists a unique $\rho_{\omega, \eta} \in \mathcal{E}$ such that

$$
\pi_{X}\left(\Phi^{t}\left(\rho_{\omega, \eta}\right)\right)=t \omega+\eta \text { for } t<-T_{0},
$$

where $\pi_{X}: T^{*} X \rightarrow X$ denotes the projection on the base manifold $X$, and where $T_{0}$ is large enough, so that $K_{0} \subset B\left(0, T_{0}\right)$. Here, $\omega$ is the incoming direction, and $\eta$ is the impact parameter. In the sequel, we will identify

$$
\left\{(\omega, \eta) ; \omega \in \mathbb{S}^{d-1}, \eta \in \omega^{\perp}\right\} \cong T^{*} \mathbb{S}^{d-1} .
$$

We define the interaction region as

$$
\mathcal{I}:=\left\{(\omega, \eta) \in T^{*} \mathbb{S}^{d-1} ; \exists t \in \mathbb{R} \text { such that } \pi_{X}\left(\Phi^{t}\left(\rho_{\omega, \eta}\right)\right) \in X_{0}\right\}
$$

By compactness of $X_{0}, \mathcal{I}$ is compact.

If $\rho_{\omega, \eta} \notin \Gamma^{-}$, then there exists $\omega^{\prime} \in \mathbb{S}^{d-1}, \eta^{\prime} \in\left(\omega^{\prime}\right)^{\perp} \subset \mathbb{R}^{d}$ and $t^{\prime} \in \mathbb{R}$ such that for all $t \geq T_{0}$,

$$
\pi_{X}\left(\Phi^{t}\left(\rho_{\omega, \eta}\right)\right)=\omega^{\prime}\left(t-t^{\prime}\right)+\eta^{\prime} .
$$

The (classical) scattering map is then defined as $\kappa(\omega, \eta)=\left(\omega^{\prime}, \eta^{\prime}\right)$, as represented on Figure 1 .

The assumptions from [GRHZ15] The main assumption in GRHZ15 is that

$$
\Gamma^{ \pm}=\emptyset \text {. }
$$

Under this assumption, $\kappa: T^{*} \mathbb{S}^{d-1} \rightarrow T^{*} \mathbb{S}^{d-1}$ is well defined. One can actually show that $\kappa$ is a symplectomorphism for the canonical symplectic structure on $T^{*} \mathbb{S}^{d-1}$, and in particular, it is invertible (see for example Gui77]).

It is easy to see that

$$
\kappa(\mathcal{I})=\mathcal{I}, \quad \kappa\left(\mathcal{I}^{c}\right)=\mathcal{I}^{c} \text { and }\left.\kappa\right|_{\mathcal{I}^{c} \rightarrow \mathcal{I}^{c}}=I d_{\mathcal{I}^{c} \rightarrow \mathcal{I}^{c}} .
$$

The results in GRHZ15] require a diversion hypothesis which concerns the periodic points of $\kappa$ in the interaction region.

For any $l \in \mathbb{Z} \backslash\{0\}$, denote the set of periodic points of $\kappa$ of period $l$ by

$$
\mathcal{F}_{l}:=\left\{(\omega, \eta) \in \mathcal{I} ; \kappa^{l}(\omega, \eta)=(\omega, \eta)\right\} .
$$




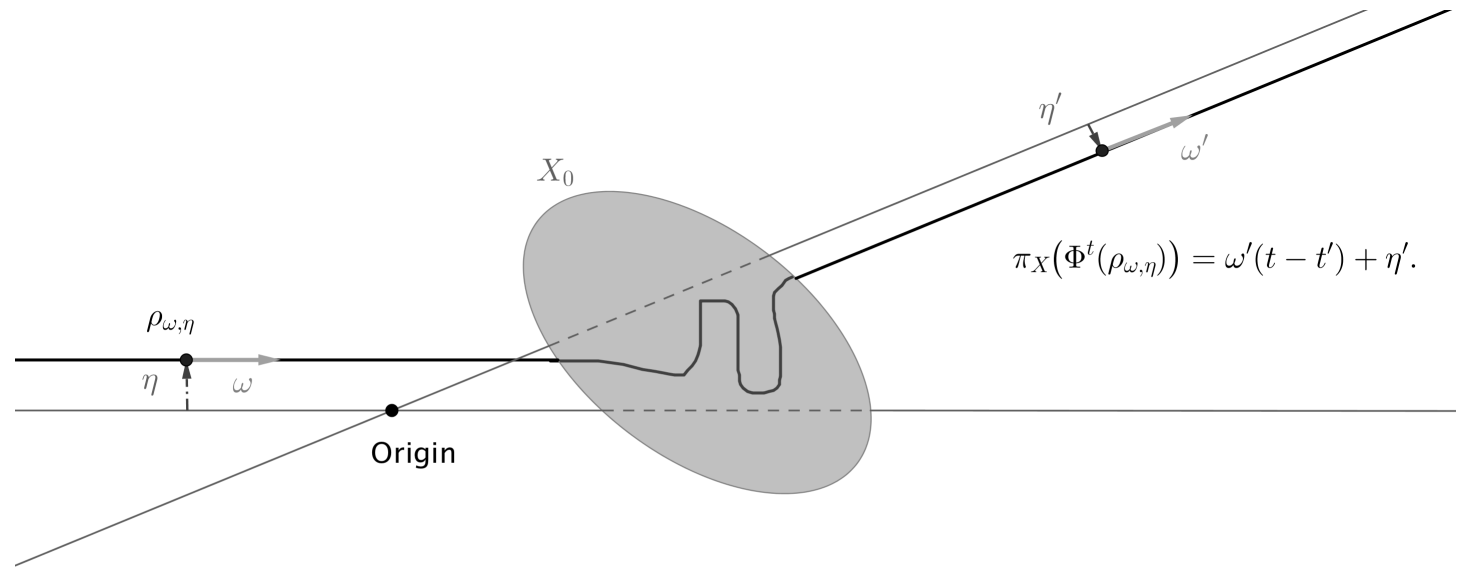

Figure 1: The scattering map $\kappa$.

The diversion hypothesis says that

$$
\forall l \in \mathbb{N} \backslash\{0\}, \quad \operatorname{Vol}\left(\mathcal{F}_{l}\right)=0,
$$

where Vol denotes the Liouville measure on $T^{*} \mathbb{S}^{d-1}$.

This hypothesis roughly says that most of the classical trajectories in $\mathcal{E}$ which interact with the potential or the perturbation of the Euclidean metric are indeed diverted. In GRHZ15, the authors work in the setting where $(X, g) \equiv\left(\mathbb{R}^{d}, g_{E u c l}\right)$, and with $X_{0}=\operatorname{supp}(V)$, and they conjecture that this hypothesis holds for generic potentials.

The main result in GRHZ15 is the following.

Theorem (GRHZ15]). Suppose that the manifold $(X, g)$ and the potential $V$ are such that (3) and (4) are satisfied. Let $f: \mathbb{S}^{1} \longrightarrow \mathbb{C}$ be a continuous function such that $1 \notin \operatorname{supp} f$. Then we have

$$
\lim _{h \rightarrow 0}\left\langle\mu_{h}, f\right\rangle=\frac{\operatorname{Vol}(\mathcal{I})}{2 \pi} \int_{0}^{2 \pi} f\left(e^{i \theta}\right) d \theta .
$$

Our objective in this paper is to show that this theorem remains true if the incoming and outgoing sets are non-empty. We define the incoming set at infinity as

$$
\tilde{\Gamma}^{-}:=\left\{(\omega, \eta) \in T^{*} \mathbb{S}^{d-1} \text { such that } \rho_{\omega, \eta} \in \Gamma^{-}\right\} .
$$

Similarly, we define the outgoing set at infinity $\tilde{\Gamma}^{+} \subset T^{*} \mathbb{S}^{d-1}$ by:

$$
\left(\omega^{\prime}, \eta^{\prime}\right) \in \tilde{\Gamma}^{+} \Leftrightarrow \exists(x, \xi) \in \Gamma^{+} ; \Phi^{t}(x, \xi)=t \omega^{\prime}+\eta^{\prime} \text { for } t \text { large enough. }
$$


Note that $\tilde{\Gamma}^{ \pm}$are compact subsets of $T^{*} \mathbb{S}^{d-1}$, since if $\eta$ is large enough, a trajectory with impact parameter $\eta$ will not meet the interaction region, and therefore cannot be trapped.

Instead of supposing (3), we will make the following assumption.

\section{Hypothesis 1.}

$$
\operatorname{Vol}\left(\tilde{\Gamma}^{ \pm}\right)=0
$$

This hypothesis is very mild: as we will see in the next section, it is satisfied as soon as the energy level $\mathcal{E}$ is non-degenerate in the sense that

$$
d p_{\mid \mathcal{E}} \neq 0 .
$$

We will also make an assumption which is the analogue of (4). In the case when (3) does not hold, this assumption is slightly more technical to write, since $\kappa^{l}$ is not well-defined on all of $\mathcal{I}$. We will therefore postpone the precise statement of this assumption to Hypothesis 2 in section 2 .

Statement of the results Under these hypotheses, we may state our result.

Theorem 1. Suppose that the manifold $(X, g)$ and the potential $V$ are such that Hypotheses 1 and 2 are satisfied. Let $f: \mathbb{S}^{1} \longrightarrow \mathbb{C}$ be a continuous function such that $1 \notin \operatorname{supp} f$. Then we have

$$
\lim _{h \rightarrow 0}\left\langle\mu_{h}, f\right\rangle=\frac{\operatorname{Vol}(\mathcal{I})}{2 \pi} \int_{0}^{2 \pi} f\left(e^{i \theta}\right) d \theta .
$$

Remark 1. For simplicity, we shall only state and prove this result for smooth potentials, but it should still be true for less regular potentials, as long as the Hamiltonian dynamics is well defined. The proof should work without many changes for a potential $V \in C_{c}^{1}(X ; \mathbb{R})$.

As in GRHZ15, we may deduce the following corollary.

Corollary 1. Let $0<\phi_{1}<\phi_{2}<2 \pi$ be angles, and let $N_{h}\left(\phi_{1}, \phi_{2}\right)$ be the number of eigenvalues $e^{i \beta_{h, n}}$ of $S_{h}$ with $\phi_{1} \leq \beta_{h, n} \leq \phi_{2}$ modulo $2 \pi$. Then we have

$$
\lim _{h \rightarrow 0}(2 \pi h)^{d-1} N_{h}\left(\phi_{1}, \phi_{2}\right)=\operatorname{Vol}(\mathcal{I}) \frac{\phi_{2}-\phi_{1}}{2 \pi} .
$$

The proof of Corollary 1 is exactly the same as that of Corollary 1.2 in [GRHZ15]: we simply approach uniformly the indicator function $\mathbf{1}_{\left[\phi_{1}, \phi_{2}\right]}$ by continuous functions and use Theorem 1 . We refer to GRHZ15 for more details.

Relation to other works The distribution of the eigenvalues of the scattering matrix has been studied since the eighties ([BY82, BY84, SY85]). More recently, in the (non-semiclassical) highenergy limit, it was studied in [BP12, and extended to more general Hamiltonians in [BP13] and [Nak14]. For related topics in the physics literature for obstacle scattering, see [DS92.

In the semiclassical setting, equidistribution of phase shifts was first observed in DGRHH14. for spherically symmetric potentials, and in GRHZ15 for more general non-trapping potentials. It was also studied in GRH15 for long-range potentials, without any assumption on the classical dynamics. In [ZZ99, the authors obtain much finer results on the distribution of phase shifts in the semiclassical limit for a family of surfaces of revolution. 
Just as in GRHZ15, the main tool in the proof of the equidistribution of phase shifts is the fact that the scattering matrix is a Fourier Integral Operator associated to the scattering map microlocally away from the incoming and outgoing directions. This was proven in Ale05, and also in HW08 in a geometric non-trapping setting.

The scattering map is trivial outside of the interaction region, while it can be very complicated inside the interaction region. This mixed behaviour is somehow similar to the situation described in MO05, where the authors prove a Weyl law for general systems for which the phase space can be separated into a part where the classical dynamics is periodic, and another where its is ergodic.

Acknowledgements The author would like to thank Stéphane Nonnenmacher for supervising this project and for useful discussions. He would also like to thank Jesse Gell-Redman, Andrew Hassell and Steve Zelditch for their comments which helped improve the first version of the manuscript. Last but not least, the author would like to thank the anonymous referee who helped improve many aspects of this paper, in particular by suggesting Lemma 1 and some of the estimates in section 5 .

The author is partially supported by the Agence Nationale de la Recherche project GeRaSic (ANR-13-BS01-0007-01).

\section{Classical dynamics}

Recall that, if $(\omega, \eta) \in T^{*} \mathbb{S}^{d-1}, \rho_{\omega, \eta}$ was defined in (2), and that we defined the sets $\tilde{\Gamma}^{ \pm}$in $(5)$ and (6).

Although we will not use it in the sequel, let us prove now the fact announced in the introduction that (8) implies Hypothesis 1. The proof is standard (it is very similar to that of [DZ, Proposition 6.5] or [GS87, Proposition A.3]), but we recall it for the reader's convenience.

Lemma 1. Suppose that $p: T^{*} X \rightarrow \mathbb{R}$ is such that $d p_{\mid \mathcal{E}} \neq 0$. Then we have $\operatorname{Vol}\left(\tilde{\Gamma}^{ \pm}\right)=0$.

Proof. Suppose that $d p_{\mid \mathcal{E}} \neq 0 . \quad \mathcal{E}$ is then a smooth manifold, which can be equipped with the Liouville measure $\mu$. This measure is invariant by the Hamiltonian flow $\left(\Phi^{t}\right)$.

Note that, outside of $X_{0}$, this measure is just the Lebesgue measure on $S^{*}\left(X \backslash X_{0}\right)$, so that, if we define for $r_{0}$ large enough and $r_{1}>r_{0}$ the annulus

$$
C_{r_{0}, r_{1}}:=\left(B\left(0, r_{1}\right) \backslash B\left(0, r_{0}\right)\right) \subset\left(\mathbb{R}^{d} \backslash K_{0}\right) \simeq\left(X \backslash X_{0}\right),
$$

we have

$$
\operatorname{Vol}\left(\tilde{\Gamma}^{ \pm}\right)=0 \Leftrightarrow \exists 0<r_{0}<r_{1} \text { large enough such that } \mu\left(\Gamma^{ \pm} \cap S^{*} C_{r_{0}, r_{1}}\right)=0 .
$$

Suppose for contradiction that we may find $0<r_{0}<r_{1}$ such that $\mu\left(\Gamma^{ \pm} \cap S^{*} C_{r_{0}, r_{1}}\right)>0$.

Since the motion of a point in $\Gamma^{ \pm} \cap S^{*} C_{r_{0}, r_{1}}$ as $\pm t \geq 0$ is just a straight line, we may find a time $t_{0}=t_{0}\left(r_{0}, r_{1}\right)$ such that for any $j \geq 1, \Phi^{ \pm j t_{0}}\left(\Gamma^{ \pm} \cap S^{*} C_{r_{0}, r_{1}}\right) \cap\left(\Gamma^{ \pm} \cap S^{*} C_{r_{0}, r_{1}}\right)=\emptyset$. Since $\Phi^{t_{0}}$ is a diffeomorphism, we then have that for all $j, j^{\prime} \in \mathbb{N}$ with $j \neq j^{\prime}$,

$$
\left(\Phi^{\mp j t_{0}}\left(\Gamma^{ \pm} \cap S^{*} C_{r_{0}, r_{1}}\right)\right) \cap\left(\Phi^{\mp j^{\prime} t_{0}}\left(\Gamma^{ \pm} \cap S^{*} C_{r_{0}, r_{1}}\right)\right)=\emptyset .
$$


Since $\mu$ is invariant by the Hamiltonian flow, we have that

$$
\begin{aligned}
\mu\left(\bigcup_{j=0}^{\infty} \Phi^{\mp j t_{0}}\left(\Gamma^{ \pm} \cap S^{*} C_{r_{0}, r_{1}}\right)\right) & =\sum_{j=0}^{\infty} \mu\left(\Phi^{\mp j t_{0}}\left(\Gamma^{ \pm} \cap S^{*} C_{r_{0}, r_{1}}\right)\right) \\
& =\sum_{j=0}^{\infty} \mu\left(\Gamma^{ \pm} \cap S^{*} C_{r_{0}, r_{1}}\right) \\
& =+\infty
\end{aligned}
$$

by assumption. But for all $j \geq 0, \Phi^{\mp j t_{0}}\left(\Gamma^{ \pm} \cap S^{*} C_{r_{0}, r_{1}}\right)$ belongs to a compact region of $\mathcal{E}$, where the base points are either in $X_{0}$, or in $B\left(0, r_{1}\right) \subset \mathbb{R}^{d}$. Hence, we must have $\mu\left(\bigcup_{j=0}^{\infty} \Phi^{\mp j t_{0}}\left(\Gamma^{ \pm} \cap\right.\right.$ $\left.\left.S^{*} C_{r_{0}, r_{1}}\right)\right)<+\infty$, a contradiction.

If $\rho_{\omega, \eta} \notin \Gamma^{-}$, then there exists $\omega^{\prime} \in \mathbb{S}^{d-1}, \eta^{\prime} \in\left(\omega^{\prime}\right)^{\perp} \subset \mathbb{R}^{d}$ and $t^{\prime} \in \mathbb{R}$ such that for all $t$ large enough,

$$
\pi_{X}\left(\Phi^{t}\left(\rho_{\omega, \eta}\right)\right)=\omega^{\prime}\left(t-t^{\prime}\right)+\eta^{\prime} .
$$

We may then define the (classical) scattering map

$$
\kappa: T^{*} \mathbb{S}^{d-1} \backslash \tilde{\Gamma}^{-} \longrightarrow T^{*} \mathbb{S}^{d-1} \backslash \tilde{\Gamma}^{+}
$$

by $\left(\omega^{\prime}, \eta^{\prime}\right)=\kappa(\omega, \eta) . \kappa$ is then a symplectomorphism.

We define the "good" sets $\left(\mathcal{G}_{k}^{+}\right) \subset T^{*} \mathbb{S}^{d-1}$ and $\left(\mathcal{G}_{k}^{-}\right) \subset T^{*} \mathbb{S}^{d-1}$ by induction for $k \in \mathbb{N}$, by

$$
\begin{array}{ll}
\mathcal{G}_{0}^{+}:=T^{*} \mathbb{S}^{d-1} \backslash \tilde{\Gamma}^{-}, & \mathcal{G}_{k+1}^{+}:=\left\{(\omega, \eta) \in \mathcal{G}_{k}^{+} \text {such that } \kappa(\omega, \eta) \in \mathcal{G}_{0}^{+}\right\} \\
\mathcal{G}_{0}^{-}:=T^{*} \mathbb{S}^{d-1} \backslash \tilde{\Gamma}^{+}, & \mathcal{G}_{k+1}^{-}:=\left\{(\omega, \eta) \in \mathcal{G}_{k}^{-} \text {such that } \kappa^{-1}(\omega, \eta) \in \mathcal{G}_{0}^{-}\right\} .
\end{array}
$$

The scattering map may then be iterated and inverted, to obtain for any $k \geq 1$ symplectomorphisms

$$
\begin{gathered}
\kappa^{k}: \mathcal{G}_{k-1}^{+} \longrightarrow \mathcal{G}_{k-1}^{-}, \\
\kappa^{-k}: \mathcal{G}_{k-1}^{-} \longrightarrow \mathcal{G}_{k-1}^{+},
\end{gathered}
$$

or, written in a more condensed way, we may define for $k \in \mathbb{Z} \backslash\{0\}, \kappa^{k}: \mathcal{G}_{|k|-1}^{\epsilon(k)} \rightarrow \mathcal{G}_{|k|-1}^{-\epsilon(k)}$, where $\epsilon(k)$ is the sign of $k$.

We also define, for $k \in \mathbb{Z} \backslash\{0\}$

$$
\mathcal{B}_{k}:=T^{*} \mathbb{S}^{d-1} \backslash \mathcal{G}_{|k|-1}^{\epsilon(k)} .
$$

$\mathcal{B}_{k}$ is hence the "bad" set where $\kappa^{k}$ is not well-defined.

Lemma 2. Suppose Hypothesis 1 is satisfied, and let $k \in \mathbb{Z} \backslash\{0\}$. Then $\mathcal{B}_{k}$ has zero Liouville measure.

Proof. By assumption, $\tilde{\Gamma}^{ \pm}$has zero Liouville measure. Since $\kappa$ preserves the Liouville measure, we see from (9) that $\mathcal{G}_{k}^{ \pm}$has full measure. 
For $l \in \mathbb{Z} \backslash\{0\}$, we define the set of $l$-periodic interacting points as

$$
\mathcal{F}_{l}:=\left\{(\omega, \eta) \in \mathcal{I} \cap \mathcal{G}_{|l|-1}^{\epsilon(l)} ; \kappa^{l}(\omega, \eta)=(\omega, \eta)\right\},
$$

where $\epsilon(l)$ is the sign of $l$. Note that this set is closed.

Our diversion hypothesis is the following.

Hypothesis 2. For any $l \in \mathbb{Z} \backslash\{0\}$, the Liouville measure of $\mathcal{F}_{l}$ is 0 .

We conjecture that if $V \equiv 0$ and if $\left(\operatorname{Int}\left(X_{0}\right), g\right.$ ) has (not uniformly) negative curvature, where $\operatorname{Int}\left(X_{0}\right)$ denotes the interior of $X_{0}$ then this hypothesis holds.

Note that, since $\kappa^{l}$ preserves the volume, this Hypothesis is equivalent to the seemingly weaker statement that for any $l \in \mathbb{N} \backslash\{0\}$, the Liouville measure of $\mathcal{F}_{l}$ is 0 .

Note also that this hypothesis implies that

$$
\operatorname{Vol}(\partial \mathcal{I})=0
$$

Indeed, a point in the boundary of $\mathcal{I}$ is in $\mathcal{I}$ because $\mathcal{I}$ is closed, and it is fixed by $\kappa$.

Before proving Theorem 1 , we need to recall a few facts and definitions from semiclassical analysis.

\section{Refresher on semiclassical analysis}

\subsection{Pseudodifferential calculus}

Let $Y$ be a compact manifold ( $Y$ will often be $\mathbb{S}^{d-1}$ in the sequel). We shall say that a function $a(x, \xi ; h) \in C^{\infty}\left(T^{*} Y \times(0,1]\right)$ is in the class $S^{c o m p}\left(T^{*} Y\right)$ if it can be written as

$$
a(x, \xi ; h)=\tilde{a}_{h}(x, \xi)+O\left(\left(\frac{h}{\langle\xi\rangle}\right)^{\infty}\right)
$$

where $\tilde{a}_{h} \in C_{c}^{\infty}\left(T^{*} Y\right)$, with $\operatorname{supp}\left(\tilde{a}_{h}\right) \subset \Omega$ for some bounded open set $\Omega$ independent of $h$, and where $\tilde{a}_{h}$ is bounded in any $C^{k}(\Omega)$ norm independently of $h$.

We associate to $S^{c o m p}\left(T^{*} Y\right)$ the algebra of pseudodifferential operators $\Psi_{h}^{c o m p}(Y)$, through a surjective quantization map

$$
O p_{h}: S^{c o m p}\left(T^{*} Y\right) \longrightarrow \Psi_{h}^{c o m p}(Y) .
$$

This quantization map is defined using coordinate charts, and the standard Weyl quantization on $\mathbb{R}^{d}$. It is therefore not intrinsic. However, the principal symbol map

$$
\sigma_{h}: \Psi_{h}^{c o m p}(Y) \longrightarrow S^{c o m p}\left(T^{*} Y\right) / h S^{c o m p}\left(T^{*} Y\right)
$$

is intrinsic, and we have

$$
\sigma_{h}(A \circ B)=\sigma_{h}(A) \sigma_{h}(B)
$$

and

$$
\sigma_{h} \circ O p: S^{c o m p}\left(T^{*} Y\right) \longrightarrow S^{c o m p}\left(T^{*} Y\right) / h S^{c o m p}\left(T^{*} Y\right)
$$

is the natural projection map. 
14].

For more details on all these maps and their construction, we refer the reader to [Wwo12, Chapter

For $a \in S^{\operatorname{comp}}\left(T^{*} Y\right)$, we say its essential support is equal to a given compact $\mathcal{K} \Subset T^{*} Y$,

$$
\operatorname{ess} \operatorname{supp}_{h} a=\mathcal{K} \Subset T^{*} Y,
$$

if and only if, for all $\chi \in C_{c}^{\infty}\left(T^{*} Y\right)$,

$$
\operatorname{supp}(\chi) \subset\left(T^{*} Y \backslash K\right) \Rightarrow \chi a \in h^{\infty} S^{\operatorname{comp}}\left(T^{*} Y\right) .
$$

For $A \in \Psi_{h}^{c o m p}(Y), A=O p_{h}(a)$, we define the wave front set of $A$ as:

$$
W F_{h}(A)=\operatorname{ess}_{\operatorname{supp}_{h}} a,
$$

noting that this definition does not depend on the choice of the quantization.

\subsection{Lagrangian states and Fourier Integral Operators}

In this section, we will recall the definition of Fourier Integral Operators with notations inspired by DG14. We refer to this paper and to the references therein for the classical proofs we omit.

Phase functions Let $\phi(y, \theta)$ be a smooth real-valued function on some open subset $U_{\phi}$ of $Y \times \mathbb{R}^{L}$, for some $L \in \mathbb{N}$. We call $x$ the base variables and $\theta$ the oscillatory variables. We say that $\phi$ is a nondegenerate phase function if the differentials $d\left(\partial_{\theta_{1}} \phi\right) \ldots d\left(\partial_{\theta_{L}} \phi\right)$ are linearly independent on the critical set

$$
C_{\phi}:=\left\{(y, \theta) ; \partial_{\theta} \phi=0\right\} \subset U_{\phi} .
$$

In this case

$$
\Lambda_{\phi}:=\left\{\left(y, \partial_{y} \phi(y, \theta)\right) ;(y, \theta) \in C_{\phi}\right\} \subset T^{*} Y
$$

is an immersed Lagrangian manifold. By shrinking the domain of $\phi$, we can make it an embedded Lagrangian manifold. We say that $\phi$ generates $\Lambda_{\phi}$.

Lagrangian states Given a phase function $\phi$ and a symbol $a \in S^{\operatorname{comp}}\left(U_{\phi}\right)$, consider the $h$ dependent family of functions

$$
u(y ; h)=h^{-L / 2} \int_{\mathbb{R}^{L}} e^{i \phi(y, \theta) / h} a(y, \theta ; h) d \theta .
$$

We call $u=(u(h))$ a Lagrangian state, (or a Lagrangian distribution) generated by $\phi$.

Definition 1. Let $\Lambda \subset T^{*} Y$ be an embedded Lagrangian submanifold. We say that an h-dependent family of functions $u(y ; h) \in C_{c}^{\infty}(Y)$ is a (compactly supported and compactly microlocalized) Lagrangian state associated to $\Lambda$, if it can be written as a sum of finitely many functions of the form (13), for different phase functions $\phi$ parametrizing open subsets of $\Lambda$, plus an $O\left(h^{\infty}\right)$ remainder in the $C^{\infty}(Y)$ topology. We will denote by $I^{\text {comp }}(\Lambda)$ the space of all such functions. 
Fourier integral operators Let $Y, Y^{\prime}$ be two manifolds of the same dimension $d$, and let $\kappa$ be a symplectomorphism from an open subset of $T^{*} Y$ to an open subset of $T^{*} Y^{\prime}$. Consider the Lagrangian

$$
\Lambda_{\kappa}=\left\{\left(y, \nu ; y^{\prime},-\nu^{\prime}\right) ; \kappa(y, \nu)=\left(y^{\prime}, \nu^{\prime}\right)\right\} \subset T^{*} Y \times T^{*} Y^{\prime}=T^{*}\left(Y \times Y^{\prime}\right) .
$$

A compactly supported operator $T: \mathcal{D}^{\prime}(Y) \rightarrow C_{c}^{\infty}\left(Y^{\prime}\right)$ is called a (semiclassical) Fourier integral operator associated to $\kappa$ if its Schwartz kernel $K_{T}\left(y, y^{\prime}\right)$ lies in $h^{-d / 2} I^{c o m p}\left(\Lambda_{\kappa}\right)$. We write $T \in$ $I^{\operatorname{comp}}(\kappa)$. Note that such an operator is automatically trace class. The $h^{-d / 2}$ factor is explained as follows: the normalization for Lagrangian states is chosen so that $\|u\|_{L^{2}} \asymp 1$, while the normalization for Fourier integral operators is chosen so that $\|T\|_{L^{2}(Y) \rightarrow L^{2}\left(Y^{\prime}\right)} \asymp 1$.

Note that if $\kappa \circ \kappa^{\prime}$ is well defined, and if $T \in I^{\operatorname{comp}}(\kappa)$ and $T^{\prime} \in I^{\operatorname{comp}}\left(\kappa^{\prime}\right)$, then $T \circ T^{\prime} \in$ $I^{\operatorname{comp}}\left(\kappa \circ \kappa^{\prime}\right)$.

The main property we will use about FIOs is the following, which is an easy version of GRHZ15, Proposition 2].

Lemma 3. Let $\kappa: T^{*} Y \supset U \rightarrow V \subset T^{*} Y$ have no fixed point, and let $T \in I^{\text {comp }}(\kappa)$. Then

$$
\operatorname{Tr}(T)=O\left(h^{\infty}\right)
$$

Proof. (Sketch) By definition, the integral kernel of $T$ can be written as a finite sum of terms of the form

$$
(2 \pi h)^{-L} \int_{\mathbb{R}^{L}} e^{i \phi\left(y, y^{\prime} ; \theta\right) / h} a\left(y, y^{\prime}, \theta, h\right) d \theta,
$$

where $\phi$ locally parametrises $\Lambda_{\kappa}$ in the sense that in some open subset $U \subset T^{*}\left(Y \times Y^{\prime}\right)$, we have

$$
\Lambda_{\kappa} \cap U=\left\{\left(y, \partial \phi_{y^{\prime}}\left(y, y^{\prime}, \theta\right), y^{\prime},-\partial_{y} \phi\left(y, y^{\prime}, \theta\right)\right) ;\left(y, y^{\prime}, \theta\right) \text { such that } \partial_{\theta} \phi\left(y, y^{\prime}, \theta\right)=0\right\} .
$$

The trace is then given by a sum of terms of the form

$$
\frac{1}{(2 \pi h)^{L+d-1}} \int_{Y} \int_{\mathbb{R}^{L}} e^{i \frac{\phi(y, y ; \theta)}{h}} a(y, y, \theta, h) d \theta d y .
$$

The fact that $\kappa$ has no fixed point implies that if $(y, y, \theta)$ are such that $\partial_{\theta} \phi(y, y, \theta)=0$, we have $\partial_{y}[\phi(y, y, \theta)]=\left[\partial_{y} \phi\left(y, y^{\prime}, \theta\right)+\partial_{y^{\prime}} \phi\left(y, y^{\prime}, \theta\right)\right]_{y=y^{\prime}} \neq 0$. Then, by non-stationary phase, we obtain the result.

\subsection{The scattering matrix as a FIO}

The main result we will use about the scattering matrix in this paper is Ale05, Theorem 5], which can be rephrased as follows.

Theorem (Alexandrova 2005). (i) Let $(\omega, \eta) \in \mathcal{G}_{0}^{+}$. If $U$ is an open neighbourhood of $(\omega, \eta)$ contained in $\mathcal{G}_{0}^{+}$and $A \in \Psi_{h}^{c o m p}\left(\mathbb{S}^{d-1}\right)$ is such that $W F_{h}(A) \subset U$, then we have $S_{h} A \in I^{\operatorname{comp}}\left(\left.\kappa\right|_{U}\right)$.

(ii) $S_{h}$ is microlocally equal to the identity away from the interaction region in the following sense. If $a \in S^{\operatorname{comp}}\left(\mathbb{S}^{d-1}\right)$ is such that $a \equiv 1$ near $\mathcal{I}$, then we have

$$
\left\|\left(S_{h}-I d\right)\left(I d-O p_{h}(a)\right)\right\|_{L^{2}\left(\mathbb{S}^{d-1}\right) \rightarrow L^{2}\left(\mathbb{S}^{d-1}\right)}=O\left(h^{\infty}\right) .
$$




\section{Trace formula}

Our aim in this section will be to prove the following proposition, which is the cornerstone of the proof in GRHZ15.

Proposition 1. Suppose that the manifold $(X, g)$ and the potential $V$ are such that Hypotheses 1 and 2 are satisfied, and let $k \in \mathbb{Z} \backslash\{0\}$. Then we have

$$
\operatorname{Tr}\left(S_{h}^{k}-I d\right)=-\frac{\operatorname{Vol}(\mathcal{I})}{(2 \pi h)^{d-1}}+o\left(h^{-(d-1)}\right) .
$$

Proof. To prove this proposition, we fix $k \in \mathbb{Z} \backslash\{0\}$, and build an adapted partition of unity.

Partition of unity Recall that $\mathcal{B}_{k}$ was defined in 10 , and is the set where $\kappa^{k}$ is not well-defined. We will write

$$
\mathcal{P}_{k}:=\mathcal{B}_{k} \cup \mathcal{F}_{k} \subset T^{*} \mathbb{S}^{d-1},
$$

where $\mathcal{F}_{k}$ are as in (11) This set is closed, has zero Liouville measure by Lemma 2 and Hypothesis 2 , and the map $\kappa^{k}$ is well-defined and has no fixed points in $\mathcal{I} \backslash \mathcal{P}_{k}$.

Since $\mathcal{P}_{k}$ is closed with zero Liouville measure, by outer regularity of the Liouville measure, we may find for each $\varepsilon>0$ a cut-off function $\chi_{\varepsilon}^{k} \in C_{c}^{\infty}\left(T^{*} \mathbb{S}^{d-1} ;[0,1]\right)$ such that $\chi_{\varepsilon}^{k}(\omega, \eta)=1$ if $(\omega, \eta) \in \mathcal{P}_{k}$, such that the support of $\chi_{\varepsilon}^{k}$ is contained in an $\varepsilon$-neighbourhood of $\mathcal{I}$, and such that the Liouville measure of the support of $\chi_{\varepsilon}^{k}$ is smaller than $\varepsilon$ :

$$
\operatorname{Vol}\left(\operatorname{supp}\left(\chi_{\varepsilon}^{k}\right)\right) \leq \varepsilon
$$

We denote by $O p_{h}\left(\chi_{\varepsilon}^{k}\right)$ the Weyl quantization of $\chi_{\varepsilon}^{k}$, as defined in section 3.1

We also take $\psi_{\varepsilon}^{1} \in C_{c}^{\infty}\left(T^{*} \mathbb{S}^{d-1} ;[0,1]\right)$ such that $\psi_{\varepsilon}^{1}=1$ near $\mathcal{I}$ and $\psi_{\varepsilon}^{1}(\omega, \eta)=0$ if $d((\omega, \eta), \mathcal{I}) \geq$ $\varepsilon$ and $\psi_{\varepsilon}^{2} \in C_{c}^{\infty}\left(T^{*} \mathbb{S}^{d-1} ;[0,1]\right)$ such that $\psi_{\varepsilon}^{2}=0$ outside of $\mathcal{I}$, and $\psi_{\varepsilon}^{2}=1$ outside of an $\varepsilon$ neighbourhood of $T^{*} \mathbb{S}^{d-1} \backslash \mathcal{I}$ (see Figure 2).

Note that we have for all $(\omega, \eta) \in T^{* \mathbb{S}^{d-1}}, \psi_{\varepsilon}^{1}(\omega, \eta) \geq \psi_{\varepsilon}^{2}(\omega, \eta)$, and that $\left\|\psi_{\varepsilon}^{1}-\psi_{\varepsilon}^{2}\right\|_{L^{1}}=O(\epsilon)$ thanks to 12 .

We have

$$
1=\left(1-\psi_{\varepsilon}^{1}\right)+\psi_{\varepsilon}^{2}\left(1-\chi_{\varepsilon}^{k}\right)+\psi_{\varepsilon}^{2} \chi_{\varepsilon}^{k}+\left(\psi_{\varepsilon}^{1}-\psi_{\varepsilon}^{2}\right) .
$$

The first term corresponds to points outside of the interaction region. The second term corresponds to points in the interaction region which are neither trapped nor fixed, while the last two terms have a support of a size $O(\varepsilon)$. We shall compute the trace of $\left(S_{h}^{k}-I d\right)$ using this decomposition.

Trace inside the interaction region By Alexandrova's Theorem (see Section 3.3), we have that $S_{h}^{k} O p_{h}\left(\psi_{\varepsilon}^{2}\left(1-\chi_{\epsilon}^{k}\right)\right)$ is a Fourier integral operator associated to $\kappa_{\left.\right|_{\mathcal{I} \backslash \mathcal{P}_{k}} ^{k}}$ microlocally near $\left(\mathcal{I} \backslash \kappa^{k}\left(\mathcal{P}_{k}\right)\right) \times$ $\left(\mathcal{I} \backslash \mathcal{P}_{k}\right)$.

Since, by definition of $\mathcal{P}_{k}, \kappa^{k}$ has no fixed points in $\mathcal{I} \backslash \mathcal{P}_{k}$, Lemma 3 tells us that

$$
\operatorname{Tr}\left(S_{h}^{k} O p_{h}\left(\psi_{\varepsilon}^{2}\left(1-\chi_{\epsilon}^{k}\right)\right)=O\left(h^{\infty}\right)\right.
$$




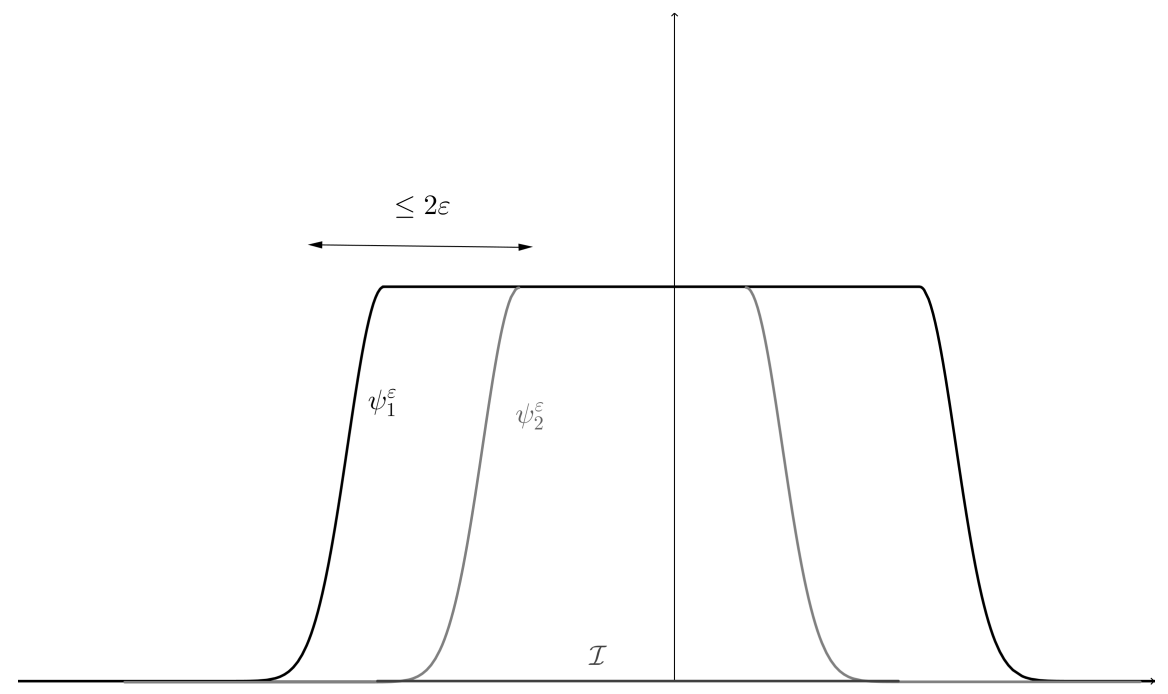

Figure 2: The cut-off functions $\psi_{1}^{\varepsilon}$ and $\psi_{2}^{\varepsilon}$

This implies that

$$
\begin{aligned}
\operatorname{Tr}\left(\left(S_{h}^{k}-I d\right) O p_{h}\left(\psi_{\varepsilon}^{2}\left(1-\chi_{\epsilon}^{k}\right)\right)\right) & =\operatorname{Tr}\left(O p_{h}\left(\psi_{\varepsilon}^{2}\left(1-\chi_{\epsilon}^{k}\right)\right)\right)+O\left(h^{\infty}\right) \\
& =\frac{1}{(2 \pi h)^{d-1}} \int_{T^{*} \mathbb{S}^{d-1}} \psi_{\varepsilon}^{2}\left(1-\chi_{\varepsilon}^{k}\right)+O\left(h^{2-d}\right) \\
& =\frac{1}{(2 \pi h)^{d-1}} \operatorname{Vol}(\mathcal{I})+h^{-(d-1)} r_{\varepsilon}+O\left(h^{2-d}\right),
\end{aligned}
$$

where $r_{\varepsilon}$ is independent of $h$, and is a $O(\varepsilon)$. To go from the first line to the second, we used the standard formula of the trace of a pseudodifferential operator as the integral of its symbol (see [Wo12, Appendix C]).

Trace outside of the interaction region To estimate the trace outside of the interaction region, we shall consider an orthonormal basis of $L^{2}\left(\mathbb{S}^{d-1}\right)$ made of spherical harmonics $\phi_{\ell}^{m}$ satisfying $\left(\Delta_{\mathbb{S}^{d-1}}-\ell(\ell+d-1)\right) \phi_{\ell}^{m}=0$, where $\ell \in \mathbb{N}, 0 \leq m \leq d_{\ell}$. Here $d_{\ell}=O\left(\ell^{d-2}\right)$, as can be seen using Weyl's law.

Let $R>0$ be large enough so that

$$
\mathcal{I} \subset\left\{(\omega, \eta) \in T^{*} \mathbb{S}^{d-1} ;|\eta| \leq R\right\} .
$$

We need the following elementary lemma:

Lemma 4. For all $R^{\prime}>R, x \in B(0, R) \subset \mathbb{R}^{d}, h>0$ and all $\ell \geq R^{\prime} / h, m \leq d_{\ell}$, we have

$$
\int_{\mathbb{S}^{d-1}} e^{i\langle\omega, x\rangle / h} \phi_{\ell}^{m}(\omega) d \omega=O\left(\left(\frac{R}{h \ell}\right)^{\infty}\right) .
$$


Proof. We have, for any $n \in \mathbb{N}$, by integration by parts,

$$
\int_{\mathbb{S}^{d-1}} e^{i\langle\omega, x\rangle / h} \phi_{\ell}^{m}(\omega) d \omega=\frac{1}{(\ell(\ell+1))^{n}} \int_{\mathbb{S}^{d-1}} \phi_{\ell}^{m}(\omega) \Delta^{n} e^{i\langle\omega, x\rangle / h} d \omega
$$

Now, $\Delta^{n} e^{i\langle\omega, x\rangle / h}$ is bounded by $\left(\frac{|x|}{h}\right)^{2 n}$ times a polynomial which depends only on $n$. The result follows.

The following lemma allows us to estimate the trace outside of the interaction region.

Lemma 5. Suppose Hypotheses 1 and 2 are satisfied, and take $k \in \mathbb{Z}$. We have

$$
\operatorname{Tr}\left(\left(S_{h}^{k}-I d\right)\left(I d-O p_{h}\left(\psi_{\varepsilon}^{1}\right)\right)\right)=O\left(h^{\infty}\right) .
$$

Proof. Let us note first that thanks to 14 , for each $\varepsilon>0, \ell \in \mathbb{N}$ and $m=1, \ldots, d_{\ell}$, we have

$$
\left\|\left(S_{h}^{k}-I d\right)\left(I d-O p_{h}\left(\psi_{\varepsilon}^{1}\right)\right) \phi_{\ell}^{m}\right\|=O\left(h^{\infty}\right) .
$$

We have

$$
\begin{aligned}
\operatorname{Tr}\left(\left(S_{h}^{k}-I d\right)\left(I d-O p_{h}\left(\psi_{\varepsilon}^{1}\right)\right)\right) & =\sum_{\ell \in \mathbb{N}} \sum_{m=1}^{d_{\ell}}\left\langle\phi_{\ell}^{m},\left(S_{h}^{k}-I d\right)\left(I d-O p_{h}\left(\psi_{\varepsilon}^{1}\right)\right) \phi_{\ell}^{m}\right\rangle \\
& =\sum_{\ell<R^{\prime} / h^{2}} \sum_{m=1}^{d_{\ell}}\left\langle\phi_{\ell}^{m},\left(S_{h}^{k}-I d\right)\left(I d-O p_{h}\left(\psi_{\varepsilon}^{1}\right)\right) \phi_{\ell}^{m}\right\rangle \\
& +\sum_{\ell \geq R^{\prime} / h^{2}} \sum_{m=1}^{d_{\ell}}\left\langle\phi_{\ell}^{m},\left(S_{h}^{k}-I d\right)\left(I d-O p_{h}\left(\psi_{\varepsilon}^{1}\right)\right) \phi_{\ell}^{m}\right\rangle \\
& =\sum_{\ell \geq R^{\prime} / h^{2}} \sum_{m=1}^{d_{\ell}}\left\langle\phi_{\ell}^{m},\left(S_{h}^{k}-I d\right) \phi_{\ell}^{m}\right\rangle+O\left(h^{\infty}\right),
\end{aligned}
$$

where $R^{\prime}>R+\varepsilon$. Here, we dealt with the sum for $\ell<R^{\prime} / h^{2}$ using 18 and the fact that $d_{\ell}=O\left(\ell^{d-2}\right)$.

Let us now bound the sum for $\ell \geq R^{\prime} / h^{2}$. Let us denote by $a_{k}\left(\omega, \omega^{\prime} ; h\right)$ the integral kernel of $S_{h}^{k}-I d$. Recall the following representation ${ }^{2}$ for $a_{1}$, which can be found in [Ale05, equation (59):

$$
a_{1}\left(\omega, \omega^{\prime} ; h\right)=c(d, h) \int_{\mathbb{R}^{d}} e^{i\langle\omega, x\rangle / h}\left(\left[h^{2} \Delta, \chi_{2}\right] R_{h}\left[h^{2} \Delta, \chi_{1}\right] e^{i\left\langle\omega^{\prime}, \cdot\right\rangle / h}\right)(x) d x,
$$

where $R_{h}=\left(P_{h}-(1+i 0)\right)^{-1}$ is the outgoing resolvent, and $\chi_{1}, \chi_{2}$ are some functions in $C_{c}^{\infty}(X)$. Here, $c(d, h):=e^{-i \pi(d-3) / 4} 2^{(-d+9) / 4}(\pi h)^{(-d+1) / 2}$ is a constant which depends polynomially in $h$. It was proven in [PZ01, §2] that the representation $[19)$ is indeed independent of the choice of the cut-off functions $\chi_{1}$ and $\chi_{2}$.

\footnotetext{
${ }^{2}$ Note that this expression for $a\left(\omega, \omega^{\prime} ; h\right)$ is smooth (and even analytic) in $\omega$ and $\omega^{\prime}$, which shows that $S_{h}-I d$ is trace-class.
} 
Now, from Bur02, Theorem 4] (see also CV02 for a more general statement, and [Dat14, Sha16] for similar statements with less regularity assumptions on $V$ ), we have that if $r_{1}>0$ is large enough, and if $r_{2}>r_{1}$, then

$$
\left\|1_{r_{1} \leq|x| \leq r_{2}} R_{h} 1_{r_{1} \leq|x| \leq r_{2}}\right\|_{L^{2}\left(\mathbb{R}^{d}\right) \rightarrow L^{2}\left(\mathbb{R}^{d}\right)}=O\left(h^{-1}\right) .
$$

From this, we get that $a_{1}\left(\omega, \omega^{\prime} ; h\right)=\int_{\mathbb{R}^{d}} e^{i\langle\omega, x\rangle / h} f_{1}\left(x, \omega^{\prime} ; h\right) d x$, where $f_{1}$ is a function which is smooth in $x$ and $\omega^{\prime}$, which is bounded polynomially in $h$, and which has support for the first variable in a compact set independent of $h$ and $\omega^{\prime}$.

Similarly, $a_{k}$ may be put in the form $a_{k}\left(\omega, \omega^{\prime} ; h\right)=\int_{\mathbb{R}^{d}} e^{i\langle\omega, x\rangle / h} f_{k}\left(x, \omega^{\prime} ; h\right) d x$, where $f_{k}$ is a function which is smooth in $x$ and $\omega^{\prime}$, which has support for the first variable in a compact set independent of $h$ and $\omega^{\prime}$, and which is bounded polynomially in $h$.

We have therefore

$$
\begin{aligned}
\left\langle\phi_{\ell}^{m},\left(S_{h}^{k}-I d\right) \phi_{\ell}^{m}\right\rangle & =\int_{\mathbb{S}^{d-1}} d \omega \int_{\mathbb{S}^{d-1}} d \omega^{\prime} \phi_{\ell}^{m}(\omega) \phi_{\ell}^{m}\left(\omega^{\prime}\right) a_{k}\left(\omega, \omega^{\prime}, h\right) \\
& =\int_{\mathbb{S}^{d-1}} d \omega^{\prime} \phi_{\ell}^{m}\left(\omega^{\prime}\right) \int_{\mathbb{R}^{d}} d x f_{k}\left(x, \omega^{\prime} ; h\right) \int_{\mathbb{S}^{d-1}} d \omega e^{i\langle\omega, x\rangle / h} \phi_{\ell}^{m}(\omega) .
\end{aligned}
$$

The last integral is bounded by $O\left(\frac{R}{h \ell^{2}}\right)^{\infty}$ thanks to Lemma 4 . Therefore, since $f_{k}$ has support for the first variable in a compact set independent of $h$ and $\omega^{\prime}$, and is bounded polynomially in $h$, we get that $\left\langle\phi_{\ell},\left(S_{h}^{k}-I d\right) \phi_{\ell}\right\rangle=O\left(\frac{R}{h \ell^{2}}\right)^{\infty}$. We may then sum this estimate over $\ell \geq R^{\prime} / h^{2}$ to get

$$
\sum_{\ell \geq R^{\prime} / h^{2}} \sum_{m=1}^{d_{\ell}}\left\langle\phi_{\ell}^{m},\left(S_{h}^{k}-I d\right) \phi_{\ell}^{m}\right\rangle=O\left(h^{\infty}\right),
$$

which concludes the proof of the lemma.

Putting it all together Thanks to equation (16), we have

$$
\begin{aligned}
\operatorname{Tr}\left(S_{h}^{k}-I d\right) & =\operatorname{Tr}\left(\left(S_{h}^{k}-I d\right)\left(I d-O p_{h}\left(\psi_{\varepsilon}^{1}\right)\right)\right) \\
& +\operatorname{Tr}\left(\left(S_{h}^{k}-I d\right) O p_{h}\left(\psi_{\varepsilon}^{2}\left(1-\chi_{\epsilon}^{k}\right)\right)\right) \\
& +\operatorname{Tr}\left(\left(S_{h}^{k}-I d\right) O p_{h}\left(\psi_{\varepsilon}^{2} \chi_{\varepsilon}^{k}+\left(\psi_{\varepsilon}^{1}-\psi_{\varepsilon}^{2}\right)\right)\right)+O\left(h^{2-d}\right) .
\end{aligned}
$$

To bound the last term, we use that

$$
\begin{aligned}
\left|\operatorname{Tr}\left(\left(S_{h}^{k}-I d\right) O p_{h}\left(\psi_{\varepsilon}^{2} \chi_{\varepsilon}^{k}+\left(\psi_{\varepsilon}^{1}-\psi_{\varepsilon}^{2}\right)\right)\right)\right| & \leq\left\|\left(S_{h}^{k}-I d\right)\right\|_{L^{2}\left(\mathbb{S}^{d-1}\right) \rightarrow L^{2}\left(\mathbb{S}^{d-1}\right)} \\
& \times\left\|O p_{h}\left(\psi_{\varepsilon}^{2} \chi_{\varepsilon}^{k}+\left(\psi_{\varepsilon}^{1}-\psi_{\varepsilon}^{2}\right)\right)\right\|_{\mathcal{L}^{1}}+O\left(h^{2-d}\right) \\
& \leq h^{-(d-1)} r_{\varepsilon}^{\prime}+O\left(h^{2-d}\right),
\end{aligned}
$$

where $r_{\varepsilon}^{\prime}$ is independent of $h$, and is a $O(\varepsilon)$.

Thanks to (17), 222 and to Lemma 5, equation 21) becomes

$$
h^{(d-1)} \operatorname{Tr}\left(S_{h}^{k}-I d\right)=\frac{\operatorname{Vol}(\mathcal{I})}{(2 \pi)^{d-1}}+r_{\varepsilon}+r_{\varepsilon}^{\prime}+O(h) .
$$

Since this is true for any $\varepsilon>0$, we obtain the statement of Proposition 1 . 
As a corollary to Proposition 1, we obtain the result for all trigonometric polynomials vanishing at 1 , that is, for any function $p$ on $\mathbb{S}^{1}$ of the form $p(z)=\sum_{-N}^{N} a_{k} z^{k}$ for some coefficients $a_{k} \in \mathbb{C}$ with $a_{0}=0$.

Corollary 2. Suppose that Hypotheses 1 and 2 are satisfied. Let $p$ be a trigonometric polynomial vanishing at 1 . Then we have

$$
\operatorname{Tr}\left(p\left(S_{h}\right)\right)=\frac{\operatorname{Vol}(\mathcal{I})}{(2 \pi h)^{d-1}} \frac{1}{2 \pi} \oint_{\mathbb{S}^{1}} p\left(e^{i \theta}\right) d \theta+o\left(h^{-(d-1)}\right) .
$$

Proof. Every trigonometric polynomial vanishing at 1 may be written as a linear combination of polynomials of the form $p(z)=z^{k}-1$, with $k \in \mathbb{Z}$, for which we have proved the result in Proposition 1.

\section{Proof of Theorem 1}

Let us define, for any $\alpha>0$,

$$
\begin{gathered}
C_{\alpha}^{0}\left(\mathbb{S}^{1}\right)=\left\{f \in C^{0}\left(\mathbb{S}^{1} ; \mathbb{C}\right) ; f(z)|\log | z-1||^{\alpha} \text { is continuous }\right\} . \\
\|f\|_{\alpha}=\sup _{|z|=1, z \neq 1}|\log | z-1||^{\alpha}|f(z)| \quad \text { for } f \in C_{\alpha}^{0}\left(\mathbb{S}^{1}\right) .
\end{gathered}
$$

Note that $C_{\alpha}^{0} \subset C_{\alpha^{\prime}}^{0}$ if $\alpha>\alpha^{\prime}$. We will now prove the following theorem, which is a slightly refined version of Theorem 1

Theorem 2. Suppose that the manifold $(X, g)$ and the potential $V$ are such that the Hypotheses 1 and 2 are satisfied. Let $\alpha>d$ and let $f \in C_{\alpha}^{0}\left(\mathbb{S}^{1}\right)$. Then we have

$$
\lim _{h \rightarrow 0}\left\langle\mu_{h}, f\right\rangle=\frac{\operatorname{Vol}(\mathcal{I})}{2 \pi} \int_{0}^{2 \pi} f\left(e^{i \theta}\right) d \theta .
$$

Before writing the proof, let us state two technical lemmas. Recall that we denote the eigenvalues of $S_{h}$ by $e^{i \beta_{n, h}}$. We shall from now on take the convention that $\left|e^{i \beta_{h, n}}-1\right| \geq\left|e^{i \beta_{h, n+1}}-1\right|$.

For any $L \geq 1$, we shall denote by $N_{L, h}$ the number of $n \in \mathbb{N}$ such that $\left|e^{i \beta_{h, n}}-1\right| \geq e^{-L / h}$.

Lemma 6. There exists $C_{0}>0$ such that for any $L \geq 1$ and $0<h<1$, we have $N_{L, h} \leq C_{0}\left(\frac{L}{h}\right)^{d-1}$

Proof. Thanks to equation (2.3) in [Chr15] (which relies on the methods developed in [Zwo89]), we have that there exists $C>0$ independent of $h$ and $n$ such that

$$
\left|e^{i \beta_{h, n}}-1\right| \leq \frac{C}{h^{d}} \exp \left(\frac{C}{h}-\frac{n^{1 /(d-1)}}{C}\right) .
$$

In particular, we have that for any $N \geq 1$,

$$
\begin{aligned}
\prod_{n=1}^{N}\left|e^{i \beta_{h, n}}-1\right| & \leq\left(\frac{C}{h^{d}}\right)^{N} \exp \left(\frac{N C}{h}-\frac{1}{C} \sum_{n=1}^{N} n^{1 /(d-1)}\right) \\
& \leq\left(\frac{C}{h^{d}}\right)^{N} \exp \left(\frac{N C}{h}-C^{\prime} N^{d /(d-1)}\right),
\end{aligned}
$$


for some $C^{\prime}>0$ independent of $h, N$.

Therefore, we have that

$$
\begin{aligned}
e^{-\frac{L N_{L, h}}{h}} & \leq \prod_{n=1}^{N_{L, h}}\left|e^{i \beta_{h, n}}-1\right| \\
& \leq\left(\frac{C}{h^{d}}\right)^{N_{L, h}} \exp \left(\frac{N_{L, h} C}{h}-C^{\prime} N_{L, h}^{d /(d-1)}\right)
\end{aligned}
$$

By taking logarithms, we get

$$
-\frac{L N_{L, h}}{h} \leq N_{L, h} \log \left(\frac{C}{h^{d}}\right)+\frac{N_{L, h} C}{h}-C^{\prime} N_{L, h}^{d /(d-1)} .
$$

The first term in the right hand side is negligible, so we get, by possibly changing slightly the constant $C^{\prime}$,

$$
C^{\prime} N_{L, h}^{d /(d-1)} \leq \frac{N_{L, h}(C+L)}{h} .
$$

Therefore, $N_{L, h} \leq\left(\frac{C+L}{C^{\prime} h}\right)^{d-1} \leq C_{0}(L / h)^{d-1}$ for some $C_{0}>0$ large enough, but independent of $L$ and $h$, which concludes the proof of the lemma.

Lemma 7. For any $\alpha>d$, there exists $C_{\alpha}>0$ such that for any $f \in C_{\alpha}^{0}\left(\mathbb{S}^{1}\right)$, we have

$$
\left|\left\langle\mu_{h}, f\right\rangle\right| \leq C\|f\|_{\alpha}
$$

Proof. We have

$$
\begin{aligned}
\left|\left\langle\mu_{h}, f\right\rangle\right| & =(2 \pi h)^{d-1}\left|\sum_{n \in \mathbb{N}} f\left(e^{i \beta_{h, n}}\right)\right| \\
& \leq(2 \pi h)^{d-1} \sum_{\left|e^{i \beta_{h, n}}-1\right| \geq e^{-1 / h}}\left|f\left(e^{i \beta_{h, n}}\right)\right|+(2 \pi h)^{d-1} \sum_{\left|e^{i \beta_{h, n}}-1\right|<e^{-1 / h}}\left|f\left(e^{i \beta_{h, n}}\right)\right| .
\end{aligned}
$$

Let us consider the first sum. By Lemma 6 , it has at most $C_{0} h^{-(d-1)}$ terms. Hence, it is bounded by

$$
(2 \pi h)^{d-1} \sum_{\left|e^{i \beta_{h, n}}-1\right| \geq e^{-1 / h}}\left|f\left(e^{i \beta_{h, n}}\right)\right| \leq(2 \pi h)^{d-1} C_{0} h^{-(d-1)}\|f\|_{C^{0}} \leq C\|f\|_{\alpha},
$$

for some $C>0$. Let us now consider the second term in (24). For each $k \geq 1$, we denote by $\sigma_{k, h}$ the set of $n \in \mathbb{N}$ such that $e^{-(k+1) / h} \leq\left|e^{i \beta_{h, n}}-1\right|<e^{-k / h}$. By Lemma $6, \sigma_{k, h}$ contains at most $C_{0}\left(\frac{k+1}{h}\right)^{d-1}$ elements. On the other hand, for each $n \in \sigma_{k, h}$, we have

$$
\left|f\left(e^{i \beta_{h, n}}\right)\right| \leq\|f\|_{\alpha}\left|\log \left(e^{-k / h}\right)\right|^{-\alpha}=\frac{h^{\alpha}\|f\|_{\alpha}}{k^{\alpha}}
$$


Therefore, we have

$$
\begin{aligned}
(2 \pi h)^{d-1} \sum_{\mid e^{i \beta_{h, n}-1 \mid<e^{-1 / h}}}\left|f\left(e^{i \beta_{h, n}}\right)\right| & =(2 \pi h)^{d-1} \sum_{k=1}^{+\infty} \sum_{n \in \sigma_{k, h}}\left|f\left(e^{i \beta_{h, n}}\right)\right| \\
& \leq(2 \pi h)^{d-1} \sum_{k=1}^{+\infty} C_{0}\left(\frac{k+1}{h}\right)^{d-1} \frac{h^{\alpha}\|f\|_{\alpha}}{k^{\alpha}} \\
& \leq C h^{\alpha}\|f\|_{\alpha},
\end{aligned}
$$

for some $C$ independent of $h$. This concludes the proof of the lemma.

Proof of Theorem 2. We have proved the result for all trigonometric polynomials vanishing at 1 in Corollary 2 Let $\alpha>\alpha^{\prime}>d$, and let $f \in C_{\alpha}^{0} \subset C_{\alpha^{\prime}}^{0}$. Let us show that $f$ can be approximated by trigonometric polynomials vanishing at 1 in the $C_{\alpha^{\prime}}^{0}$ norm, which will conclude the proof of the theorem thanks to Lemma 7.

Since $f(z)\left(1+|\log | z-1||^{2 \alpha}\right)^{1 / 2}$ is continuous, we may find a sequence $P_{n}$ of polynomials such that

$$
\left\|P_{n}-f(z)\left(1+|\log | z-1||^{2 \alpha}\right)^{1 / 2}\right\|_{C^{0}} \leq 1 / n
$$

Since $f(0)=0$, we may suppose that $P_{n}(1)=0$. We may also suppose that $P_{n}^{\prime}(1)=0$ (for a proof of this fact, see for example Dur12, Theorem $8, \S 6]$ ).

Since the function $|\log | z-1||^{\alpha^{\prime}}\left(1+|\log | z-1||^{2 \alpha}\right)^{-1 / 2}$ is continuous, we have that

$$
\left\|P_{n}|\log | z-1||^{\alpha^{\prime}}\left(1+|\log | z-1||^{2 \alpha}\right)^{-1 / 2}-f(z)|\log | z-1||^{\alpha^{\prime}}\right\|_{C^{0}} \leq C / n .
$$

Now, since $P_{n}(1)=P_{n}^{\prime}(1)=0$, the function $P_{n} /\left((z-1)\left(1+|\log | z-1||^{2 \alpha}\right)^{1 / 2}\right)$ is continuous, and we may find a polynomial $Q_{n}$ such that

$$
\left\|\frac{P_{n}}{(z-1)\left(1+|\log | z-1||^{2 \alpha}\right)^{1 / 2}}-Q_{n}\right\|_{C^{0}} \leq 1 / n
$$

Since the function $(z-1)|\log | z-1||^{\alpha^{\prime}}$ is continuous, we obtain that

$$
\left\|P_{n}|\log | z-1||^{\alpha^{\prime}}\left(1+|\log | z-1||^{2 \alpha}\right)^{-1 / 2}-Q_{n}(z-1)|\log | z-1||^{\alpha^{\prime}}\right\|_{C^{0}} \leq C^{\prime} / n
$$

Combining (26) and (27), we obtain that $f$ can be approached by $(z-1) Q_{n}$ in the $C_{\alpha^{\prime}}^{0}$ norm. This concludes the proof of Theorem 2

\section{References}

[Ale05] I. Alexandrova. Structure of the semi-classical amplitude for general scattering relations. Comm. Partial Differential Equations, 30(10-12):1505-1235, 2005.

[BP12] D. Bulger and A. Pushnitski. The spectral density of the scattering matrix for high energies. Communications in Mathematical Physics, 316, Issue 3.:693-704, 2012. 
[BP13] D. Bulger and A. Pushnitski. The spectral density of the scattering matrix of magnetic schrödinger operator for high energies. J. Spectr. Theory, 3, Issue 4.:517-534, 2013.

[Bur02] N. Burq. Lower bounds for shape resonances width of long range Schrödinger operators. Amer. J. Math., 124(4):677-735, 2002.

[BY82] M. Sh. Birman and D. R. Yafaev. Asymptotic behavior of the limiting phase shifts in the case of scattering by a potential without spherical symmetry. Theoretical and Mathematical Physics, 51(1):344-350, 1982.

[BY84] M. Sh. Birman and D. R. Yafaev. Asymptotic behavior of the spectrum of the scattering matrix. Journal of Soviet Mathematics, 25(1):793-814, 1984.

[Chr15] T.J. Christiansen. A sharp lower bound for a resonance-counting function in even dimensions. arXiv preprint arXiv:1510.04952, 2015.

[CV02] F. Cardoso and G. Vodev. Uniform estimates of the resolvent of the laplace-beltrami operator on infinite volume riemannian manifolds with cusps. ii. Annales Henri Poincaré, 3(4):673-691, 2002.

[Dat14] K. Datchev. Quantitative limiting absorption principle in the semiclassical limit. Geometric and Functional Analysis, 24(3):740-747, 2014.

[DG14] S. Dyatlov and C. Guillarmou. Microlocal limits of plane waves and Eisenstein functions. Ann. Sci. Éc. Norm. Sup, 47(2):371-448, 2014.

[DGRHH14] K. Datchev, J. Gell-Redman, A. Hassell, and P. Humphries. Approximation and equidistribution of phase shifts: spherical symmetry. Communications in Mathematical Physics, 326, Issue 3.:209-236, 2014.

[DS92] E. Doron and U. Smilansky. Semiclassical quantization of chaotic billiards: a scattering theory approach. Nonlinearity, 5:1055-1084, 1992.

[Dur12] P. L. Duren. Invitation to classical analysis, volume 17. American Mathematical Soc., 2012.

[DZ] S. Dyatlov and M. Zworski. Mathematical theory of scattering resonances. Version 0.03, To appear.

[GRH15] J. Gell-Redman and A. Hassell. The distribution of phase shifts for semiclassical potentials with polynomial decay. arXiv preprint 1509.03468, 2015.

[GRHZ15] J. Gell-Redman, A. Hassell, and S. Zelditch. Equidistribution of phase shifts in semiclassical potential scattering. Journal of the London Mathematical Society, 91(1):159179, 2015.

[GS87] C. Gérard and J. Sjöstrand. Semiclassical resonances generated by a closed trajectory of hyperbolic type. Communications in Mathematical Physics, 108(3):391-421, 1987.

[Gui77] V. Guillemin. Sojourn times and asymptotic properties of the scattering matrix. Publications of the Research Institute for Mathematical Sciences, 12(Supplement):6988, 1977. 
[HW08] A. Hassell and J. Wunsch. The semiclassical resolvent and the propagator for nontrapping scattering metrics. Adv. Math., 217(2):586-682, 2008.

[Mel95] R.B. Melrose. Geometric Scattering Theory. Cambridge University Press, 0995.

[MO05] J. Marklof and S. O'Keefe. Weyl's law and quantum ergodicity for maps with divided phase space, with an appendix by S. Zelditch. Nonlinearity, 18:277-304, 2005.

[Nak14] S. Nakamura. Microlocal properties of scattering matrices. arXiv preprint 1407.8299, 2014.

[PZ01] V. Petkov and M. Zworski. Semi-classical estimates on the scattering determinant. Annales Henri Poincaré, 2(4):675-711, 2001.

[Sha16] J. Shapiro. Semiclassical resolvent bounds in dimension two. arXiv preprint arXiv:1604.03852, 2016.

[SY85] A. V. Sobolev and D. R. Yafaev. Phase analysis in the problem of scattering by a radial potential. Zapiski Nauchnykh Seminarov POMI, 147:155-178, 1985.

[Zwo89] M. Zworski. Sharp polynomial bounds on the number of scattering poles. Duke Math. $J, 59(2): 311-323,1989$.

[Zwo12] M. Zworski. Semiclassical Analysis. AMS, 2012.

[ZZ99] S. Zelditch and M. Zworski. Spacing between phase shifts in a simple scattering problem. Communications in mathematical physics, 204(3):709-729, 1999. 